\title{
The Impact of COVID-19 on the Relation Between Retail Investors' Trading and Stock Returns in the Chinese Market
}

\author{
Tao Bing ${ }^{1}$ \\ 1 School of Finance, Capital University of Economics and Business, China \\ Keywords: chinese stock market, positive feedback trading, retail investors, covid-19 \\ 10.46557/001c.19015
}

Asian Economics Letters

Vol. 2, Issue 1, 2021

\begin{abstract}
This paper investigates the relation between retail investors' flows and returns during the COVID-19 pandemic in the Chinese market using the VAR model. The results show that though the positive feedback trading during the pandemic is weaker than that in the pre-COVID-19 period, the positive feedback trading following negative returns during the crisis is much stronger than that in the pre-COVID-19 period. This implies panic trading.
\end{abstract}

\section{Introduction}

The COVID-19 pandemic has led to serious repercussions for financial market performance. This literature covering the effect of COVID-19 on the stock market (Gil-Alana \& Claudio-Quiroga, 2020; Salisu \& Sikiru, 2020), market sentiment (Baig et al., 2020; Huang \& Zheng, 2020), firm performance (Fu \& Shen, 2020; Polemis \& Soursou, 2020), among others, is rich and evolving. However, little is known about investors' behavior during this pandemic. The exceptions are Ortmann et al. (2020) and Glossner et al. (2020), who investigate the behavior of retail investors and institutions in the UK and the US, respectively. Given that the Chinese market is dominated by retail investors and their trades have greater price impact, especially during crisis periods (see Burch et al., 2016), it is important to understand the retail investors' behaviors in the Chinese market during the COVID-19 pandemic.

This paper explores retail investors' behavior and their forecastability during the COVID-19 pandemic by analyzing the relation between retail investors' flows and returns. The main findings are as follows. First, there exists significant retail investors' positive feedback trading in the preCOVID-19 period and during the COVID-19 crisis. We find that the positive feedback trading is stronger in the preCOVID-19 period. Further analysis shows that the positive feedback trading following positive returns in the preCOVID-19 period becomes negligible and insignificant during the COVID-19 pandemic. On the other hand, the positive feedback trading following negative returns during the COVID-19 pandemic is stronger than that in the preCOVID-19 period.

Second, retail investors' net flows are negatively correlated with future returns both in the pre-COVID-19 period and during the COVID-19 crisis. We find that the negative relation is stronger during the COVID-19 crisis.
These findings make two contributions to the literature. First, our paper extends the literature on the relation between investors' flows and returns by analyzing the change of retail investors' feedback trading and its forecastability during the COVID-19 pandemic in the world's largest emerging market, namely, the Chinese stock market. Second, the finding that the stronger positive feedback trading following negative returns during the crisis provides novel idea about the pricing power of the rise-favor asymmetric feedback trading factor in Chinese market provided by Wan et al. (2016).

\section{Data and Methodology}

The transaction data are obtained from the WIND database. According to the classification criteria of orders, there are four types of orders: small order, medium order, large order and extra-large order, corresponding to the single transaction amounts less than 40 thousand RMB, 40-200 thousand RMB, 0.2-1 million RMB and greater than 1 million RMB, respectively. Generally, small and medium orders are regarded trading from retail investors, whereas others are from institutions. The net flows of retail investors (NBV_Retail) are their purchases minus sales (Samarakoon, 2009; and Vo, 2017). To test the robustness of the results, only the small orders will be used as the proxy to calculate the flow of retail investors $\left(N B V_{-} S\right)$. The returns (RetZZQZ) are calculated using the whole market index (CSI All Share Index) compiled by the China Securities Index Company Limited.

As the comparison of the relation between retail investors' flows and returns before and during the COVID-19 pandemic is the focus of this paper, the sample period from 01/01/2019 to 31/03/2020 is covered. On 31 December 2019, the WHO China Country Office was informed of cases of pneumonia of unknown etiology. Since then the infected 
cases have been published every day. Thus, 31/12/2019 will be chosen as the start of the COVID-19 crisis. Further, on March 31, 2020, workers from Wuhan with qualified nucleic acid test were allowed to return to their working provinces, indicating that the pandemic in China was under control. Therefore, 31/03/2020 will be chosen as the end date of the COVID-19 crisis. The pre-COVID-19 sample covers the period form 01/01/2019 till 30/12/2019.

The summary statistics and the augmented Dickey-Fuller unit root tests results are presented in Table 1. The results show that all the series are stationary.

To explore the relationship between retail investors' trading and returns, the bivariate vector autoregressive (VAR) model is as follows:

$$
\left\{\begin{array}{c}
N B V \_ \text {Retail }{ }_{t}=\alpha_{0} \\
+\sum_{j=1}^{4} \alpha_{1 j} N B V \_ \text {Retail }_{t-j} \\
+\sum_{j=1}^{4} \alpha_{2 j} \operatorname{Ret} Z Z Q Z_{t-j}+\varepsilon_{1 t} \\
\operatorname{Ret} Z Z Q Z_{t}=\beta_{0} \\
+\sum_{j=0}^{4} \beta_{1 j} N B V \_ \text {Retail }_{t-j} \\
+\sum_{j=1}^{4} \beta_{2 j} \operatorname{Ret} Z Z Q Z_{t-j}+\varepsilon_{2 t}
\end{array}\right.
$$

where $\alpha_{0}$ and $\beta_{0}$ are the constants; $N B V \_$Retail $t_{t}$ is the flow of retail investors on day $t ; \operatorname{Ret} Z Z Q Z_{t}$ is the market return on day $t$. We consider the contemporaneous impact by assuming $\beta_{0} \neq 0$ is considered. The optimal lag order of four is determined after applying the Akaike Information Criterion (AIC).

\section{Results \\ A. Retail Investors' feedback trading behavior}

Figure 1 depicts the net flows of retail investors to a shock in returns in the pre-COVID-19 period and during the COVID-19 crisis. The first graph in Fig. 1 shows that retail investors display significant positive feedback trading in the two periods. Moreover, in the pre-COVID-19 period, the positive feedback trading is stronger than during the COVID-19 period. This is consistent the notion that the irrational trading by lots of retail investors significantly influences the Chinese market (see Wan et al., 2016).

The potential asymmetries in retail investors' positive feedback trading following positive returns (Rpos) and negative returns (Rneg) are further investigated. The results are shown in the second and third plots in Figure 1.

We find that there is no asymmetry in the two periods: in the pre-COVID-19 period, the positive feedback trading following the positive and negative returns are both significant, but they are insignificant during the COVID-19 period.

By comparison, over the two periods, we find significant differences. In the pre-COVID-19 period, the positive feedback trading following positive returns is stronger than that following negative returns. This behavior is consistent with the finding of Wan et al. (2016), who show that the differ- ence between the positive feedback trading to positive and negative returns is a new pricing factor in the Chinese market, and this is the result of the herding behavior of irrational retail investors. However, the feedback trading following positive returns during the COVID-19 crisis becomes negligible and insignificant, whereas the positive feedback trading following negative returns is stronger than that in the pre-COVID-19 period. During the crisis, the positive feedback trading following negative returns is much stronger. This may reflect the panic trading behavior in retail investors due to the COVID-19 outbreak. In addition, the different intensity of the positive feedback trading following positive and negative returns during the crisis provides important implications for the pricing power of the new factor.

\section{B. The impact of net flows on returns}

Figure 2 depicts the impulse responses of returns to net flows of retail investors. The first graph in Fig. 2 shows that retail investors' net flows are negatively correlated with future returns both before and after the COVID-19 outbreak. The contemporaneous relations are negative and persistent. This indicates that their poor market timing of retail investors is the result of their information disadvantage instead of the price pressure. This result provides direct evidence of loss for retail investors, consistent with the retail investors' irrational positive feedback trading as explained earlier. Besides, the negative relation is stronger during the COVID-19 pandemic, which confirms existence of panic trading.

We further investigate potential asymmetries in the response of returns to retail investors' buying and selling. The results are shown in the second and third plots in Figure 2. The results show significant asymmetry in both the two periods: the positive return following sales of retail investors is significant in the contemporaneous day over the next several days, whereas the negative return's response to buying is negligible and insignificant. This suggests that the loss for retail investors is mainly because of their poor market timing in selling. Moreover, during the COVID-19 pandemic, the timing in selling is worse, which explains the stronger negative relation in the first plot in Figure 2.

\section{Robustness tests}

To ensure the robustness of our results, we replace retail investors' flows with small orders. To save space, the results are not shown here, but they are available on request from the author. Overall, the results suggest the above findings are robust in terms of significance, amplitude and sign of the impulse response, except the impact of retail investors' buying on returns before the COVID-19 outbreak. In fact, the impulse response of returns to buying of small orders is significantly negative, whereas that for the sum of small orders and medium orders is negligible and insignificant. This result further indicates the bad timing of buying for retail investors. 
Table 1: Summary statistics and the results of Augmented Dickey-Fuller unit root tests

Panels A: Summary statistics

\begin{tabular}{|c|c|c|c|c|c|c|c|c|c|c|}
\hline \multirow[b]{2}{*}{ Variable } & \multicolumn{5}{|c|}{ Before the COVID-19 outbreak } & \multicolumn{5}{|c|}{ During the COVID-19 pandemic } \\
\hline & Mean & St.Dev. & Min & Max & $\mathrm{N}$ & Mean & St.Dev. & Min & Max & $\mathrm{N}$ \\
\hline$N B V_{-} S$ & 16.064 & 15.547 & -19.998 & 77.718 & 233 & 28.032 & 24.012 & -12.69 & 95.93 & 59 \\
\hline NBV_Retail & 16.316 & 18.744 & -30.989 & 80.807 & 233 & 30.873 & 30.866 & -22.32 & 118.6 & 59 \\
\hline RetZZQZ & 0.001 & 0.013 & -0.067 & 0.056 & 233 & -0.001 & 0.020 & -0.0820 & 0.0360 & 59 \\
\hline \multicolumn{11}{|c|}{ Panels B: Results of Augmented Dickey-Fuller unit root tests } \\
\hline & \multicolumn{5}{|c|}{ Before the COVID-19 outbreak } & \multicolumn{5}{|c|}{ During the COVID-19 pandemic } \\
\hline NBV_Retail & \multicolumn{5}{|c|}{$-11.911^{* * *}$} & \multicolumn{5}{|c|}{$-8.396^{* * *}$} \\
\hline RetZZQZ & \multicolumn{5}{|c|}{$-14.666^{* * *}$} & \multicolumn{5}{|c|}{$-7.583^{* * *}$} \\
\hline
\end{tabular}

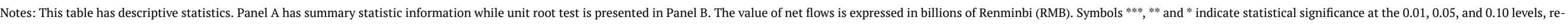
spectively. 

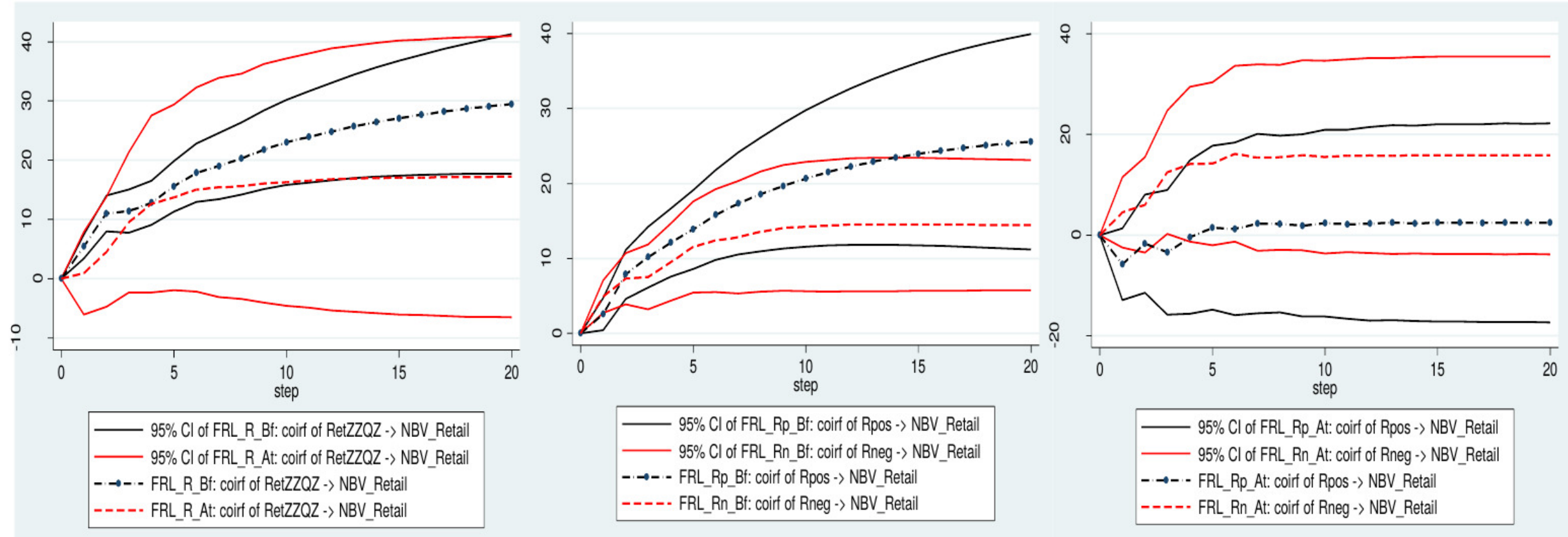

Figure 1: Cumulative impulse responses of retail investors' net flows to market returns, and positive and negative returns.

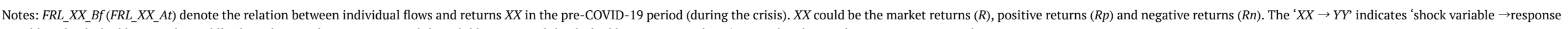
variable'. The dashed lines in the middle show the impulse responses, and the solid lines around the dashed line represent the $95 \%$ error bands. 0 is the contemporaneous day. 


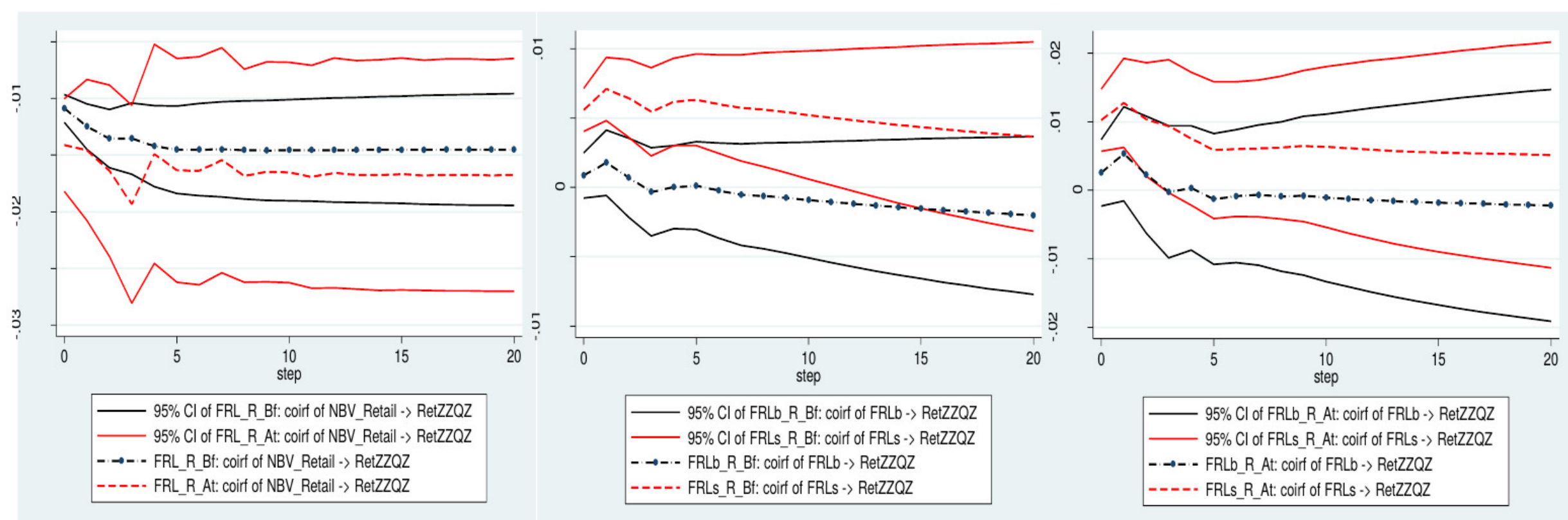

Figure 2: Cumulative impulse responses of returns to investors' net flows, buying and selling.

Notes: $F R L b$ (FRLS) denote the buying (selling) of retail investors. 


\section{Conclusions}

The relation between retail investors' flows and returns during the COVID-19 pandemic in the Chinese stock market has been explored in this paper using the VAR model. The results show that the positive feedback trading during the COVID-19 period is weaker than that in the pre-COVID-19 period. We find that the positive feedback trading following negative returns during the COVID-19 is stronger than that in the pre-COVID-19 period, indicating the panic displayed by retail investors during the crisis.

Submitted: December 03, 2020 AEDT, Accepted: January 29, 2021 AEDT

This is an open-access article distributed under the terms of the Creative Commons Attribution 4.0 International License (CCBY-SA-4.0). View this license's legal deed at https://creativecommons.org/licenses/by-sa/4.0 and legal code at https://creativecommons.org/licenses/by-sa/4.0/legalcode for more information. 


\section{REFERENCES}

Baig, A. S., Butt, H. A., Haroon, O., \& Rizvi, S. A. R. (2020). Deaths, panic, lockdowns and US equity markets: The case of COVID-19 pandemic. Finance Research Letters, 38, 101701. https://doi.org/10.1016/ j.frl.2020.101701

Burch, T. R., Emery, D. R., \& Fuerst, M. E. (2016). Who Moves Markets in a Sudden Marketwide Crisis? Evidence from 9/11. Journal of Financial \& Quantitative Analysis, 51(2). https://doi.org/10.1016/ j.ribaf.2020.101240

Fu, M., \& Shen, H. (2020). COVID-19 and Corporate Performance in the Energy Industry. Energy Research Letters, 1(1). https://doi.org/10.46557/001c.12967

Gil-Alana, L. A., \& Claudio-Quiroga, G. (2020). The COVID-19 IMPACT on the ASIAN STOCK MARKETS. Asian Economics Letters, 1(2). https://doi.org/10.4655 7/001c.17656

Glossner, S., Matos, P., Ramelli, S., \& Wagner, A. F. (2020). Where Do Institutional Investors Seek Shelter when Disaster Strikes? Evidence from COVID-19 (No. DP15070). [Working Paper]. https://ssrn.com/abstrac $\mathrm{t}=3655271$

Huang, W., \& Zheng, Y. (2020). COVID-19: Structural changes in the relationship between investor sentiment and crude oil futures price. Energy Research Letters, 1(2), 13685. https://doi.org/10.46557/001c.13 $\underline{685}$
Ortmann, R., Pelster, M., \& Wengerek, S. T. (2020). COVID-19 and investor behavior. Finance Research Letters, 37, 101717. https://doi.org/10.1016/j.frl.202 $\underline{0.101717}$

Polemis, M., \& Soursou, S. (2020). Assessing the Impact of the COVID-19 Pandemic on the Greek Energy Firms: An Event Study Analysis. Energy Research Letters, 1(3). https://doi.org/10.46557/001c.1 $\underline{7238}$

Salisu, A. A., \& Sikiru, A. A. (2020). Pandemics and the Asia-Pacific Islamic Stocks. Asian Economics Letters, 1(1). https://doi.org/10.46557/001c.17413

Samarakoon, L. P. (2009). The relation between trades of domestic and foreign investors and stock returns in Sri Lanka. Journal of International Financial Markets, Institutions and Money, 19(5), 850-861. https://doi.or g/10.1016/j.intfin.2009.07.001

Vo, X. V. (2017). Trading of foreign investors and stock returns in an emerging market - Evidence from Vietnam. International Review of Financial Analysis, 52, 88-93. https://doi.org/10.1016/j.irfa.2017.05.007

Wan, D., Liu, W., Wang, J., \& Yang, X. (2016). Asymmetries of Positive Feedback Trading in Individual Stocks: Evidences from China. Journal of Management Science and Engineering, 1(1), 3-27. http s://doi.org/10.3724/sp.j.1383.101002 